\title{
Scalable Back-Bone-Assisted Hop Greedy Routing for VANETS
}

\author{
Rucha Patil \\ PG Student, Dept of Computer Engineering, SSBT's College of Engineering and Technology, North Maharashtra \\ University, Jalgaon, Maharashtra, India
}

\begin{abstract}
In variety of application such as information sharing, navigation system, internet access vehicular area network plays an important role. To broadcast message safely, optimal routing we need more research. In such a case efficient routing plays an important role. To reach to destination we use shortest path algorithm but it can gives higher hop count. By adding mobile nodes to ad hoc network, it will deviate the path far from optimal path because it has no knowledge current topology which cause higher hop count. In this paper we propose optimize routing technique for VANET which improve routing by continuously monitoring and change the path which causes less hop count.
\end{abstract}

Keywords: Vehicular ad -hoc network (VANET), optimal path, hop count, ExBAHG.

\section{INTRODUCTION}

Vehicle to infrastructure and vehicle to vehicle communication is provided by VANET. It is a highly dynamic topology. More research is required in the routing, vehicular sensing, safety message broadcast etc. In a network we can't predict the actual traffic density. The most connected road path will be overcrowded by sending packet on same path by considering connectivity parameter. In this paper we propose an algorithm based on both hop count as well as connectivity. The propose protocol provides lower energy, higher throughput, lower latency. In this design we divided the map into different zones. And the boundary condition will be the intersection as shown in figure 1

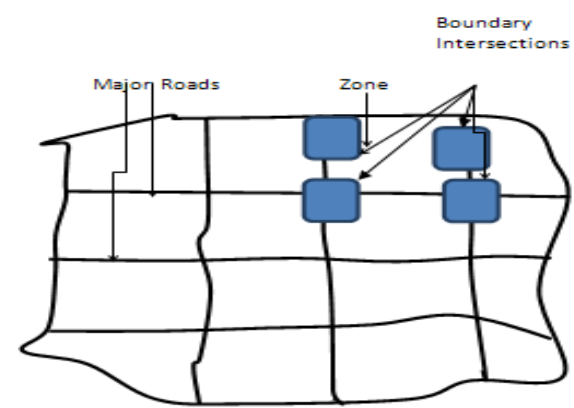

Figure1: Zone structure

In this figure we divide the map into 16 polygon, this are called zones. The connectivity is increase by increasing number of lanes; Major roads having more than two lanes are together form a polygon. Boundary intersection is present at the corner of each zone. Connectivity should be preserve at road segment and at intersection .To maintain connectivity backbone nodes are use. Backbone node at intersection is show in figure 2.

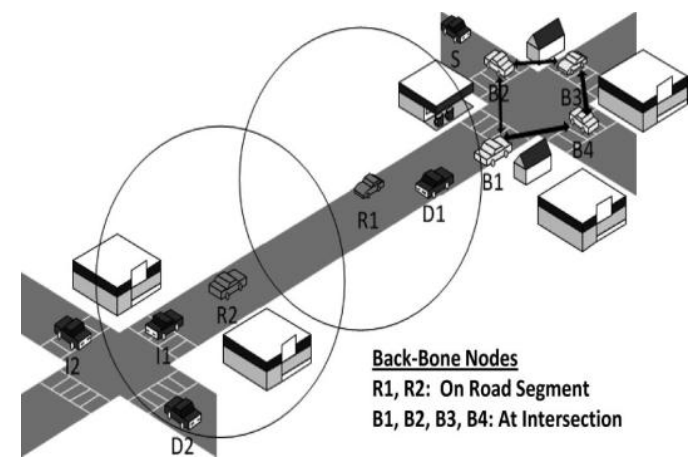

Figure 2 Backbone node structure 
Vol. 6, Issue 6, June 2017

There are three types of backbone node stable, primary, secondary. The node which is waiting at an intersection is called stable. The node which are crossing the intersection, at the intersection primary node is located and outside the intersection secondary backbone is located. Secondary backbone is chosen by primary backbone by considering direction of vehicle, speed and position.

\section{Motivation}

The existing shortest path algorithm are prone to find the shortest path and less hop count. During this we face some of the following problem:

- $\quad$ Location Service Requirement Problem

As the information has to travel a number of hop in the area, retrieving the real time position of source and destination is nearly impossible. In hierarchical location service (HLS) and grid location service (GLS) lower beacon interval is the key factor for higher accuracy. Computational and communication overhead is included in aggregation and disseminating the position information throughout the entire network. For every movement new path is required from the intermediate intersection so hop count will be increased.

\section{- $\quad$ Intersection Node Probing Problem}

Intersection plays an important role in data communication. Due to small intersection region the probability of change in direction is very high. Choosing an unstable node as a forwarding node will be risky. Some protocols like GPSR and GSR do not gives connectivity hence it causes serious impact on their performance.

\section{- $\quad$ Distance or Connectivity Based Weighted Graph Problem}

Node is consider as vertices when ad-hoc network scenario is mapped into graph weights are calculated using the Euclidean distance between the end point. As their is presence of higher buildings vehicles are unable to communicate if Euclidean distance between them is less than transmission range. As the distance between source and destination is less but the hop count is increases.

\section{LITERATURE SURVEY}

Literature survey plays vital role in the software development process. Different time factor, economic factor are taken into consideration.

The content of the paper focuses on the research and contributions of various sources. These include:

[1] The paper describe real time vehicular traffic information protocol which requires information to create the road base path. This paper will overcome this problem also avoid the location service problem.

[2] The paper describes destination discovery oriented routing in the VANET but their is no dedicated path .This paper will provide the technique to find the dedicated path and find the minimal path for the nodes.

\section{IMPLEMENTATION}

Implementation plays a vital role in the project development. At this stage, the project is converted in to actual working system. Careful planning, study of existing system also plays an important role in implementation phase.

Implementation of the proposed system involves the environment in which the system is implemented and the overall system development. The overall development of the proposed system requires suitable environment and proper resources for its successful completion.

The source and destination are continuously moving so their will be chances of another shortest path to move which provide less hop count. To find the optimal path with less hop count we use ExBAHG algorithm. Packet header will include the hop count. Whenever the packet is taken hop count will be incremented by one at the source it will be zero $(\mathrm{HC}=0)$. The hop comparison array include (SA,DA,HC,NA) where SA is source address , DA is destination address, HC is Hop count, NA is neighbours address from which packer is broadcasted. Rest of the algorithm is explained below.

\section{Algorithm 1}

When node receives the packet $P$

BEGIN

1. IF the node a is the final destination address consumes the packet GOTO END;

2. (Assume P belongs to (SAk, DAk greater than flow) ) Compare SAk, DAk to all the valid entries in the hop comparison array;

3. IF there is no match with the entries ,store (SAk, DAk, HCk, NAk) in the hop comparison 
array;

4. IF the packet is destined to a as the next hop node, process the packet for forwarding further.

GOTO END

5. (Assume that it matched with an entry ( $\mathrm{SAk}, \mathrm{DAk}, \mathrm{HCj}, \mathrm{NAj}$ )

IF ( HCk - HCj greater than 2) a short cut is found node a does the following :

5.1 send a message to NAj to update the routing table such a that the next hop address for the destination node DAk is modified to address of the node a.

5.2 DAk will be modified as NAk in its routing table by making next-Hop address fo destination DAk as NAk;

\section{END}

5.3 Modify the hop comparison array, delete the entry corresponding to ( SAk,DAk ) ;

Algorithm 1.Parh Aware Algorithm to find the optimal path

The proposed system provides better result as compared to the existing system.

\section{RESULTS}

The average end to end delay against source and destination distance is shown in figure 3(a). In graph the comparison between BAHG[3] and SAHG is shown, previously delay is same but BAHGs goes on increasing as the distance between source and destination increases but SAHG gives good results as it checks again is there any another shortest path is shown in figure 3(b). End-to-End Delay against Packet sending Rate. End-to-End Delay against Destination Dislocation Distance is shown in figure 3(c).

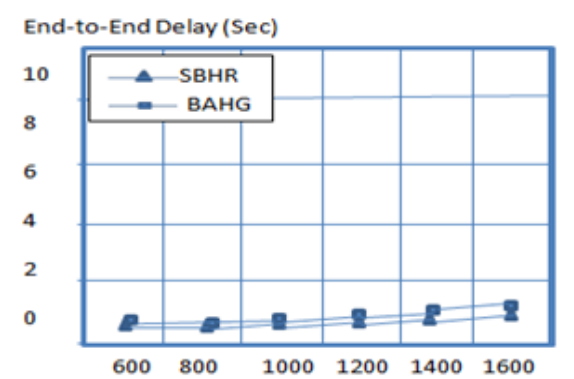

figure 3(a) . Initial source Destination Distance (meters)

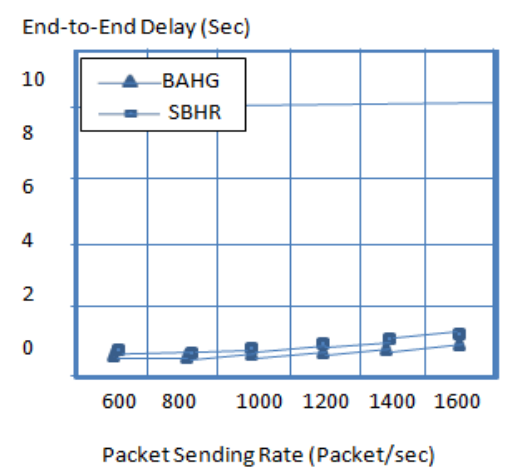

figure3(b). Packet sending rate (packet/sec)

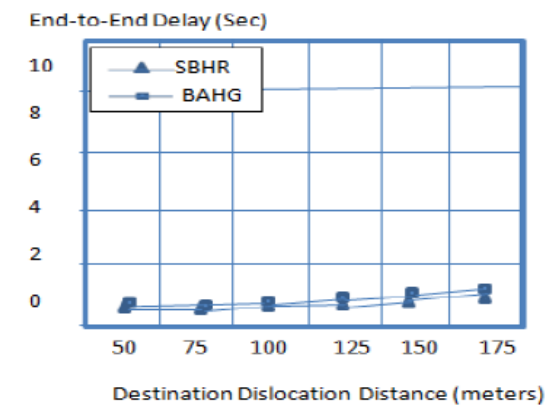

figure 3(c). Destination Dislocation Distance (meters) 
The Result will provides better reliability, integrity and security as compared to the existing system.

\section{VI .CONCLUSION}

The intersection node probing problem is covered in ExBAHG by using backbone node, as well as optimizing the routing technique for vehicular ad-hoc network. By monitoring routing path continuously ExBAHG improves routing optimality.

\section{REFERENCES}

[1] J.Nzouonta,et al, "VANET routing on city road using real-time vehicular traffic information", IEEE Trans. Veh. Technol., 58, no. 7, pp.36093626, Sep. 2008.

[2] Pratap Kumar Sahu, Eric Hsiao-Kuang Wu, Jagruti Sahoo, and Mario Gerla," BAHG:

[3] Back-Bone-Assisted Hop Greedy Routing for VANET City Environments", IEEE TRANSACTIONS ON INTELLIGENT TRANSPORTATION SYSTEMS, VOL. 14, NO. 1 ,

[4] MARCH 2013.

[5] K. C. Lee, J. Herri, U. Lee, and M. Gerla, "Enhanced perimeter routing for geographic forwarding protocols in urban vehicular scenarios", in Proc. IEEE Globe Com Workshops, 2007, pp. 110.

[6] G. Liu, B. S. Lee, B. C. Seet, C. H. Foh, K. J.Wong, and K. K. Lee," A routing strategy for metropolis vehicular communications", in Proc. ICOIN, LNCS, Aug. 2004, pp. 134143.

[7] M. Jerbi, S.-M. Senouci, R. Meraihi, and G.-D. Yacine," An improved vehicular Ad-hoc routing protocol for city environments", Reviewed at the Direction of IEEE International Conference on Communications, pp. 3972 3979, 24-28 June 2007.

[8] B. Karp and H. T. Kung, "GPSR: Greedy perimeter stateless routing for wireless networks," in Proc. ACM MOBICOM, 2000, pp. 243-254

[9] P. K. Sahu, E. H. Wu, J. Sahoo, and M. Gerla, "DDOR: Destination discovery oriented routing in highway/freeway VANETs," in Springer Telecommun. Syst.-Special Issue Vehicular Communications, Networks, Applications, 2010, pp. 1-18. 